\title{
Microscopylnnovations
}

\section{Microscopy Today Innovation Awards}

The editors of Microscopy Today congratulate the winners of the seventh Microscopy Today Innovation Award competition. The ten innovations described below advance microscopy in several areas: light microscopy, scanning probe microscopy, electron microscopy, and hybrid microscopy-analysis methods. These innovations will make microscopy and microanalysis more powerful, more flexible, more productive, and easier to accomplish.

\section{Hard X-ray Scanning Microscope with Nanofocusing Optics}

\section{Brookhaven National Laboratory and Argonne National Laboratory}

Developers: Evgeny Nazaretski, Yong Chu, Kenneth Lauer, Hanfei Yan, Brian Mullany, Nathalie Bouet, Juan Zhou, Dennis Kuhne, and Deming Shu

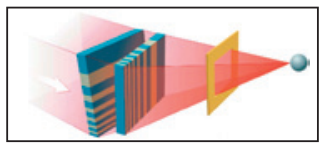

In this new hard X-ray microscope, monochromatic X-rays are focused to produce a nanoscale-size beam. X-ray imaging is performed by scanning a sample under the nanofocused beam while using various techniques to collect elemental, structural, and chemical information from the sample. Previously Fresnel zone plates, refractive lenses, and elliptical mirrors were used to produce nanofocused X-ray beams. This instrument employs a new class of X-ray nanofocusing optics, known as Multilayer Laue Lenses (MLL), to achieve imaging with sub- $20 \mathrm{~nm}$ spatial resolution. A pair of MLL linear optics, oriented 90 degrees to each other, is employed to produce a small spot of $\mathrm{X}$ rays. To achieve nanometer-scale resolution, alignment of these optics requires an ultra-stable piezo-based positioning system coupled with active interferometric feedback control on eight axes. This combination of positioning control provides a vibrational stability of less than $2 \mathrm{~nm}$ at all frequencies and long-term thermal drifts better than $2 \mathrm{~nm} /$ hour. A typical focal distance for MLL optics at $12 \mathrm{keV}$ is a few millimeters, resulting in a working distance of about $1 \mathrm{~mm}$.

The MLL microscope is an in-vacuum system that serves as a general purpose $\mathrm{X}$-ray microscopy tool suitable for a broad range of imaging experiments. The following techniques are supported: X-ray fluorescence, ptychography, diffraction, differential phase contrast, and X-ray absorption spectroscopy. The system is installed on the Hard X-ray Nanoprobe (HXN) beamline at the National Synchrotron Light Source-II (NSLS-II) facility at Brookhaven National Laboratory in Upton, NY.

This system is the first multi-purpose MLL microscope with resolution below $20 \mathrm{~nm}$ that can provide simultaneous multimodality in terms of elemental, structural, and chemical variations across the sample. The multimodality of the scanning hard X-ray microscope allows users to perform comprehensive structural and chemical studies across both the physical and biological sciences. For example, the hierarchical architecture of a chromosome was determined without the sectioning that would be required for conventional transmission electron microscopy (TEM) techniques. The MLL-based X-ray microscopy system serves as the primary end-station at the HXN beamline at NSLS-II. It is open to the general user community and supports imaging experiments spanning many fields-materials science, chemistry, and biology.

\section{X-Cite ${ }^{\circledR}$ TURBO with LaserLED Hybrid Drive ${ }^{T M}$}

\section{Excelitas Technologies}

Developers: Paul Constantinou, Yong Wang, Yu Song, K. Babu, and Tom Porter

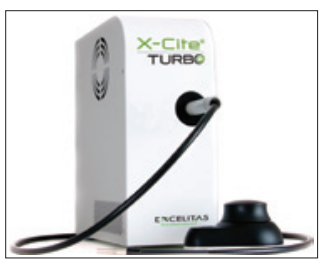

The X-Cite ${ }^{\circledR}$ TURBO is a solid-state illuminator for fluorescence microscopy that can supply six individually controllable wavelengths covering the UV and visible spectra.

Discrete, high-power LEDs generate five of X-Cite TURBO's six wavelengths. To overcome the limitation of LEDs in the $500 \mathrm{~nm}$ to $600 \mathrm{~nm}$ region of the spectrum, known as the LED green gap, a novel laser-pumped phosphor technique is used. High-efficiency blue lasers excite a phosphor layer, generating a broad peak from $525 \mathrm{~nm}$ to $625 \mathrm{~nm}$, which can then be filtered to a more specific excitation band depending on the fluorophore of interest. With more power in the $525-625 \mathrm{~nm}$ region, the exposure time required to obtain a good fluorescence signal is dramatically reduced.

Other schemes using blue LEDs or lasers in combination with a phosphor have been introduced to help overcome the LED green gap, but previous technologies do not use the phosphor to its full potential. The X-Cite TURBO optical system is optimized for illumination uniformity and maximum light delivery at all wavelengths. The ability of the laser to be focused on the phosphor layer allows the LaserLED Hybrid Drive to minimize the generation of unusable excess light, thereby increasing the coupling efficiency to a microscope's optical system. Not only does this reduce the energy required to generate a given amount of excitation light, it reduces the thermal load on the phosphor. This simplifies the cooling infrastructure required to maintain optical stability and maximizes light output over time. In addition, feedback sensors are available in some models of the product to allow continuous control and monitoring of the light output. Color and spectral 


\section{Microscopy of America Awards}

Nominations are now open for the Microscopy Society of America Annual Awards. The awards process is one way in which the Microscopy Society of America recognizes the significant and diverse contributions that individuals make to our field. Deserving nominations for consideration should be submitted electronically no later than October 31st, 2016 to:

\section{AssociationManagement@microscopy.org}

\section{The Main Society Awards Are}

\section{Distinguished Scientist Awards}

These Awards recognize preeminent senior scientists from both the Biological and Physical disciplines who have a long-standing record of achievement during their career in the field of microscopy or microanalysis.

\section{Burton Medal}

The Burton Medal was initiated to honor the distinguished contributions to the field of microscopy and microanalysis of a scientist who is less than 40 years of age on January 1 st of the award year. (Please note the change in the selection criterion regarding age.)

\section{Outstanding Technologist Awards}

These Awards honor technologists from both the Biological (Hildegard $\mathrm{H}$. Crowley Award) and Physical Sciences (Chuck Fiori Award) who have made significant contributions such as the development of new techniques which have contributed to the advancement of microscopy and microanalysis.

\section{Morton D. Maser Distinguished Service Award}

This Award was initiated to recognize outstanding volunteer service to the Society as exemplified by Mort Maser, who served the Society for many years with great dedication. This award is made to honor an MSA member who has provided significant volunteer service to the Society over a period of years.

\section{The Albert Crewe Award}

The Albert Crewe Award was initiated to recognize the distinguished contributions to the field of microscopy and microanalysis in the physical sciences of a postdoctoral fellow of not more than 6 years' standing (since doctoral graduation).

\section{The George Palade Award}

The George Palade Award was initiated to recognize the distinguished contributions to the field of microscopy and microanalysis in the life sciences of a postdoctoral fellow of not more than 6 years' standing (since doctoral graduation).
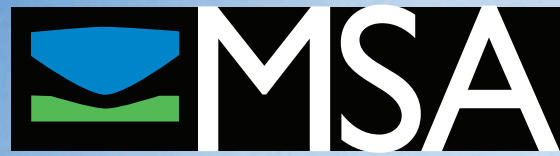

Microscopy Society of America
Further details of the nomination process can be found on the society webpage at: www.microscopy.org 
control are achieved by combining and balancing specific wavelength components individually.

There is growing demand for non-lamp (mercury, xenon) based light engines for applications such as fluorescence excitation and endoscopy. With many research institutions looking to phase out mercury-based systems entirely, a solid-state replacement is becoming more important. The environmental benefits include reduced energy consumption and elimination of harmful mercury waste. Moreover, this is not just a replacement technology, this innovation is also an enabling technology for the growing number of analytical instruments designed for rapid, high-throughput processing of biological samples.

\section{Leica TCS SP8 DLS Confocal Microscope}

\section{Lecia Microsystems}

Developers: Werner Knebel, Daniel Unholtz, Wernher Fouquet, Petra Haas, Frank Sieckmann, and Sebastian Hitzler

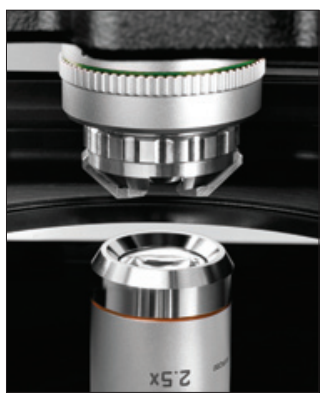

This new microscope combines light sheet microscopy and confocal microscopy in one system without compromising either functionality. It allows the combination of the two methods, for example, confocal photomanipulation with subsequent light sheet acquisition. Light sheet fluorescent microscopy (LSFM) reduces phototoxic effects and fluorophore bleaching by illuminating a specimen in only a single plane at a time. With the system's sCMOS cameras, image acquisition is fast. Therefore, LSFM is a useful tool for studying fast dynamic processes in sensitive living cells.

A typical light sheet microscope has a perpendicular setup where the illuminating and the detection objectives are arranged at a $90^{\circ}$ angle to each other. The Leica TCS SP8 DLS turns LSFM vertically by using a mirror device that deflects the light sheet at $90^{\circ}$. The transmitted light arm of the confocal microscope is replaced by detection optics featuring an objective lens and a high-speed camera. This approach enables retrofitting of light sheet functionality onto an already-installed platform.

The sample can be manipulated in the confocal imaging mode before it is imaged by LSFM. For example, a photoconvertible fluorescent protein can be activated in a subpopulation of cells in the confocal mode, and subsequently the behavior of these specific cells can be investigated over longer time periods by light sheet microscopy. Inherent in the design of this system, the user has a fully functional confocal microscope, which also can be equipped with other advanced technologies like STED superresolution, multiphoton excitation, etc. Any visible laser used for confocal microscopy, including a flexible white-light laser, can also be used as an excitation source for light sheet imaging.

Quality fluorescence images are always a trade-off between fluorophore bleaching and sufficient signal from the specimen. In recent years, light sheet microscopy has emerged as a valuable tool for imaging of living organisms. Light sheet imaging is fast and gentle, and hence helpful when observing sensitive samples or fast biological processes. This means that samples can be observed over extremely long time periods without losing the signal or impairing the specimens. As an example application, LSFM has become the method of choice for the observation of developing embryos in real-time.

\section{Nanojehm SEM Aura Work Station}

\section{Nanojehm, Inc.}

Developers: Eric Lifshin, Mathew Zotta, Matt Berkoetter, Yukun Han, and David Frey

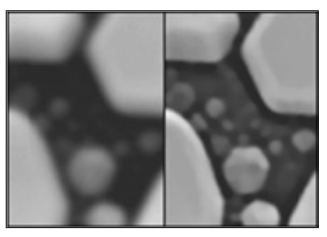

This software-based system uses deconvolution and regularization methods to sharpen images from any SEM. The process is simple. The microscopist first takes an image of the calibration standard, and the Nanojehm Aura Workstation determines the spatial distribution of electrons in the beam, that is, the point spread function (PSF). The user then collects one or more images of a sample, and the software corrects for drift and vibration while ensuring good statistical sampling. Finally, the advanced computational capability of the workstation applies deconvolution and regularization methods to rapidly sharpen each image. The result is an image with improved resolution and better overall quality.

Using this process, thermionic-source instruments have produced sharp images at greater than $200,000 \times$, a magnification normally associated with field emission gun SEMs. This system allows sharp images to be produced with larger-than-usual electron probes. For thermionic instruments, the probe current increases dramatically with probe size. Thus, high-quality images can be obtained several times faster than those obtained with small probes that have far less current. This method is also useful for low-voltage images where the probe size is larger than at higher voltages. In this case, improved resolution is possible without the need for beam deceleration. Another benefit of the PSF determination step is that astigmatism can be rapidly detected indicating the status of the instrument or whether adequate corrections have been made.

Over the years there has been much confusion about just what probe size and resolution actually mean. The Nanojehm PSF determination step provides what may be the best method yet to accurately determine probe size, and in the case of axially symmetric probes the information can be applied to accurately characterize the probe diameter as that containing a fixed percentage (say $90 \%$ ) of the probe current.

The Nanojehm approach has been used to successfully improve overall thermionic SEM performance (for SE, BS, and STEM images), low-voltage performance for both thermionic and FEG SEMs, and imaging situations where long working distances and thus larger probes may be encountered. Opportunities in nanotechnology, microelectronic and micromechanical devices, and nanobiology reveal a need for high-resolution imaging on less expensive SEMs. 


\section{G-mode Microscopy and Spectroscopy}

\section{Oak Ridge National Laboratory}

Developers: Alex Belianinov, Suhas Somnath, Liam F. Collins, Sergei V. Kalinin, and Stephen Jesse

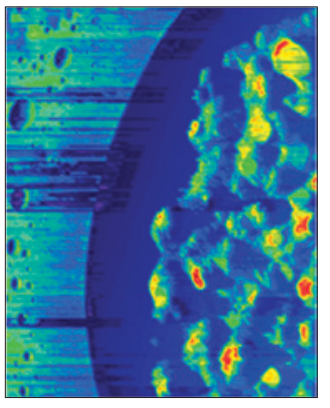

G-mode imaging and spectroscopy acquires the full scanning probe response at the maximal bandwidth limit of the detector that is followed by multivariate analysis of the complete data set. This approach replaces classical heterodyne-based detection in single- and dual-frequency scanning probe microscopes (SPMs) including lock-in amplifiers, phase-locked loop systems and their derivatives like dual amplitude resonance tracking (DART), multiple simultaneous lock-in based methods, and parallel detection methods such as Band Excitation. G-mode is universally applicable to all dynamic SPM modes (topography, magnetic, mechanical, etc.).

In G-mode, the storage and lossless compression of the full information flow from the detector allow the user to examine all relevant aspects of the probe response. Moreover, information-theory-based analytics can be used to visualize dynamic behavior, detect rare events, decouple mixed signals, etc. In many cases, the data can be constrained using physical models to completely characterize the details of tip-surface interaction. This information is not available in heterodyne techniques employed in most of the 50,000 SPM platforms operating worldwide. G-mode enables spectroscopic imaging 100-1000 thousand times faster than the current state-of-art by combining full data acquisition and adaptive filtering with physics-based reconstruction. In spectroscopy, improvements in the measurement speed, subsequent drift reduction, and adjustable spatial resolution enable complex multidimensional experiments capable of untangling the interplay of material properties at the nanoscale. Results by this new method can always be reduced to the typical SPM data obtained with traditional methods.

G-mode detection can be implemented in existing tools with minimal effort and investment because the required components are already built in as a part of the typical heterodyne detection scheme. As a result, this method unlocks new imaging and data interpretive capabilities without the need to update and configure new, expensive hardware (beyond additional G-mode electronics).

G-mode is applicable to the entire suite of AFM measurements such as Kelvin probe, magnetic force, piezoresponse force, and many other microscopies. From a purely experimental perspective, visualization of material properties with the ability to tune the interplay between the microscope resolution, acquisition time, and resampling of the data permits an entirely new way to think about and differentiate processes occurring at the tip-surface junction.

\section{SciScan}

\section{Scientifica, Ltd}

Developers: Bruno Pichler, Arjun Prakash, Alex Murray, and Dale Elgar

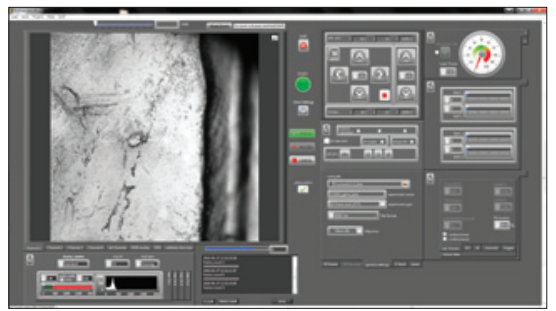

SciScan software is an open-source package for the control of laser scanning microscopes (LSMs). Although its primary application is to acquire imaging data with Scientifica's multiphoton microscopes, SciScan can be used for image acquisition with any LSM employing National Instruments DAQ hardware. This includes the majority of custom-built multiphoton microscopes and many commercial third-party systems. SciScan is provided free of charge to the scientific community, independent of hardware sales.

Similar to a number of other LSM software packages, SciScan allows users to control the microscope and acquire images directly. What sets SciScan apart from other commercial LSM software packages is that it not only provides an internal scripting language (SciScript) to set up complex acquisition schemes, but it also has an ActiveX-based application program interface (API) for external scripting. This API provides access to the full set of SciScan functions from any software that allows ActiveX communication. Virtually all commonly used data analysis and programming environments, such as MATLAB, Python, $\mathrm{C}++$, and Java, offer this type of ActiveX support.

SciScan makes it possible for a researcher to control image acquisition from prepared scripts and thereby integrate SciScan into the same environments used for the processing and analysis of the data, as well as for figure or report generation. Thus, SciScan directly connects data acquisition with the data pipeline or workflow, allowing full automation of the entire process. The interoperability of SciScan with existing data pipelines is further enhanced by the support of the Open Microscopy Environment (OME). This facility allows any OME-capable software to load SciScan data and metadata without the need for proprietary import filters. Experiments are easily scalable, allowing a single user to fully automate data acquisition on multiple systems at the same time, enabling higher throughput of data acquisition and opening the door for "big data" applications.

The scriptability, scalability, and openness of SciScan make it an ideal match for structured research initiatives where largescale data are acquired by multiple users aiming to build vast databases of biomedical information. For example, the Allen Brain Institute in Seattle is already leveraging the versatility of SciScan's internal and external scripting to facilitate their novel "Structured Science" approach. 


\section{Everstill $^{\mathrm{TM}} \mathrm{K}-400$}

\section{Technical Manufacturing Corporation}

\section{Developers: Tony Lopes and Emil Kraner}

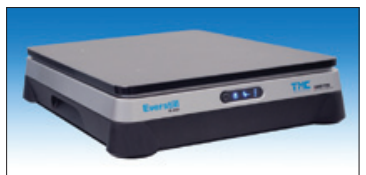

The Everstill ${ }^{\mathrm{TM}} \mathrm{K}-400$ is a new active inertial vibration cancelation (VC) system. This patented technology delivers aggressive vibration cancelation starting at $0.7 \mathrm{~Hz}$. The system provides true "inertial" active vibration (AV) cancelation. This means the isolation system includes vibration, not position, sensors that continuously measure mechanical vibration with respect to the Earth and actuators in a closedloop feedback system that act with an opposing force to cancel the measured vibration. In addition, the Everstill K-400 employs a serial-type active VC system where the sensor-actuator is in series with a spring. Only such a serial-type inertial active VC design can provide highly aggressive low-frequency (below $10 \mathrm{~Hz}$ ) vibration cancelation.

In the Everstill K-400, the active bandwidth starts at $0.7 \mathrm{~Hz}$ and continues up to $150 \mathrm{~Hz}$, delivering $50 \%$ cancelation at $1 \mathrm{~Hz}$ and $90 \%$ starting at $2 \mathrm{~Hz}$, with the active bandwidth extending out to $100 \mathrm{~Hz}$. This is achievable because of two key design features. First, the active control loop is decoupled from the payload. This allows a large bandwidth, in spite of payload resonances, without the need for payload-dependent tuning, retuning, or even de-tuning as is the case with parallel-type technologies. Second, velocity-type sensors, with sensitivity down to sub- $1 \mathrm{~Hz}$ levels, are combined with high-capacity actuators for exceptional low-frequency vibration cancelation. To achieve the aggressive attenuation that Everstill delivers, a velocity-type sensor is required. The signal from the absolute velocity sensor is amplified and then processed via sophisticated algorithms before it is sent to the actuators to counteract the floor or table vibration starting at approximately $0.7 \mathrm{~Hz}$.

Existing bench-top vibration isolation solutions use passive springs, self-leveling pneumatic passive isolators, or alternative active vibration cancelation. These solutions are inadequate in the $0.7 \mathrm{~Hz}$ to $10 \mathrm{~Hz}$ region. In addition these solutions amplify vibration at their resonances, which are typically in the 1.5 to $5 \mathrm{~Hz}$ range.

The main use of the Everstill K-400 is as a support for light microscopes, scanning probe microscopes, gravitometers, and other benchtop metrology instruments on existing lab tables. Applications include, but are not limited to, in-vitro fertilization, digital microscopy, live cell imaging, and atomic force microscopy (AFM) for molecular and protein investigation.

\section{Lynx EVO}

\section{Vision Engineering Inc.}

\section{Developers: Vision Engineering Ltd}

The Lynx EVO is an eyepiece-less stereo microscope providing exceptional ergonomic performance for intricate inspection and manipulation tasks requiring $3 \mathrm{D}$ (stereo) imaging at magnifications up to $240 \times$. This microscope employs Vision Engineering's patented Dynascope ${ }^{\circledR}$ eyepiece-less

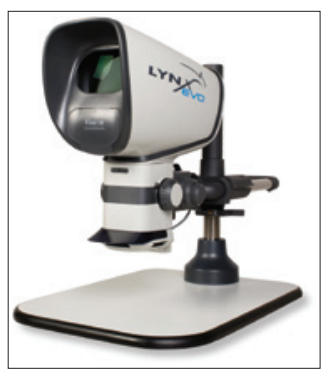

optical projection technology. Dynascope technology removes the need for conventional microscope eyepieces, liberating the user from a fixed body posture resulting in a more comfortable and efficient user experience.

At the heart of the Dynascope ${ }^{\circledR}$ eyepiece-less technology is a spinning multi-lenticular disc. On the surface of the disk are more than 3.5 million individual lenses (known as lenticules) that act as independent image-forming surfaces. Each individual lens has a diameter of just a few micrometers. The multi-lenticular disk spins at high speed to merge the millions of individual optical paths into a high-clarity image. Light reflects from the Dynascope optics, exiting the single viewing lens as twin (stereo) light paths. The large diameter of these exit rays (approximately $30 \mathrm{~mm}$ ) means that users do not need to precisely align their eyes with the viewing lens in order to see the image because the exit pupils are projected out from the head of the viewing window. This arrangement does away with interpupillary distance adjustment, allowing spectacle wearers to keep their spectacles on. In addition, this technology gives the user freedom of head movement. Users can move within the optical path to effectively look around a subject without moving it.

The Lynx EVO optical specifications include a 10:1 zoom ratio with long working distances that provide users with inspection magnifications of up to $120 \times(6 \times-60 \times$ standard $)$, ideal for a wide range of applications. With the addition of a multiplier module, magnification can be increased to $240 \times$ without reducing the working distance, extending the effective zoom ratio up to 20:1. Systems can be adapted with a range of options to suit individual applications, including various stands, $\mathrm{HD}$ image capture, and a $360^{\circ}$ rotating viewer accessory that provides an all-around angled view of the subject. Examples of applications include inspection of microelectronics assemblies, sample preparation for other analysis methods, and inspection of medical equipment such as blades, stents, and catheters.

\section{Speckle-Free Semiconductor Laser}

\section{Yale University}

Developers: Brandon Redding, Alex Cerjan, Xue Huang, Larry Lee, Douglas Stone, Michael Choma, and Hui Cao

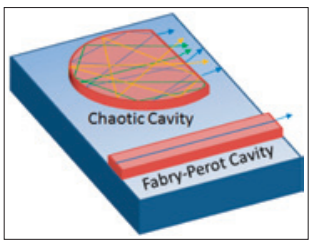

High spatial coherence is a defining characteristic of laser emission. While spatial coherence is advantageous in many applications, it can also produce artifacts such as the speckle that has precluded the use of lasers in most full-field imaging applications. As a result, low-spatial coherence sources such as thermal sources and light emitting diodes (LEDs) remain dominant in most imaging systems. However, these sources lack the brightness required for next-generation high-speed imaging systems. 
To employ a laser in full-field imaging applications, the speckle effect must be eliminated. Although methods to suppress speckle exist (for example, rotating diffusers), these solutions require long exposure times to average over many speckle patterns, limiting the ability to perform high-speed imaging. To address this, we developed a multimode semiconductor laser capable of producing low-spatial coherence emission for speckle-free imaging. Specifically, we optimized a chaotic cavity design to obtain highly multimode lasing by minimizing competition. The design was then fabricated experimentally on a commercially available GaAs laser diode wafer. A standard Fabry-Perot cavity was fabricated on the same chip for comparison. Experimentally, we found that the chaotic cavity laser supported $\sim 1000$ lasing modes. In contrast, the standard Fabry-Perot laser supported only a few modes because of strong mode competition. In a simple imaging test, we confirmed that the multimode emission from the chaotic cavity laser indeed produced speckle-free images while illumination from the Fabry-Perot cavity laser resulted in the speckle typical of traditional lasers. In addition to providing specklefree emission, the chaotic cavity laser emission was four orders of magnitude brighter (higher power per mode) than an LED. The chaotic cavity laser provides an $8 \mathrm{~nm}$ bandwidth (centered at $\sim 800 \mathrm{~nm})$.

This innovation enables direct full-field imaging, in contrast to alternative approaches such as swept-source optical coherence tomography or laser scanning microscopy. The current prototype can be used for full-field confocal microscopy, but extension of the chaotic cavity design to the visible spectrum could enable such applications as an optical pump in full-field fluorescence microscopy. Thus, by combining the brightness of a laser with the low-spatial coherence of an LED in a compact, low-cost platform, the chaotic cavity laser could enable a wide range of high-speed full-field imaging applications.

\section{Zeiss Stemi 305 with Labscope}

\section{Carl Zeiss Microscopy GmbH}

Developers: Patrick Nagel, Steven Wan, Halley Gu, Eva Huang, Ralph Jin, Sherry Xu, Ralph Aschenbach, Guangmei Wu, Walter Song, Jonee Li, Peter Kraemer, Benno Radt, Kerstin Hofmann, Axel Laschke, Joerg Sprenger, and Thorsten Wehr

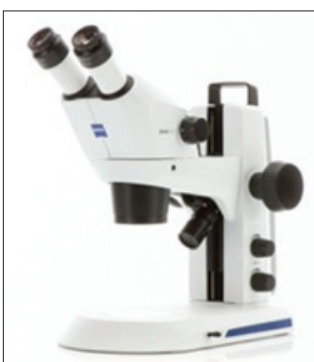

The Zeiss Stemi 305 cam in conjunction with the supporting application, Zeiss Labscope, forms a Wi-Fi-enabled microscope network, establishing an integrated imaging system that allows easy documentation, measurement, and analysis as well as the sharing of information locally or through the Internet. The Stemi 305 cam is a compact Greenough stereo microscope dedicated to biology classroom and laboratory use as well as industrial inspection applications. It integrates the microscope stand with various illumination methods. A color 1.2-megapixel Wi-Fi camera and router are integrated into the microscope body. For stand-alone use, each Stemi 305 cam can deliver its own Wi-Fi net and can connect with up to six Apple iPads. To create a digital classroom, multiple Stemi 305 cam microscopes may be connected to the same existing $\mathrm{Wi}-\mathrm{Fi}$ network.

Zeiss Labscope, the Zeiss imaging app, connects to the Stemi 305 cam stereo microscope Wi-Fi network. Any number of microscopes can be accessed from a single iPad, iPhone, or PC without the need for configuration or fiddling with IP addresses. Either an isolated microscope network or an existing institutional Wi-Fi network may be used.

The Zeiss imaging app Labscope streams the live images of all Stemi 305 cam microscopes in the network to each connected iPad, iPhone, or PC. With one touch the teacher can look at each student's results. It's easy to snap images, add annotations and measurements, and save them locally-or export them directly to the shared Windows network folder. Once connected to the internet you can share images, reports, or videos with others via email, social media, or cloud services. The Zeiss Stemi 305 cam with Labscope Wi-Fi-based microscope network is easy to install and nearly cable-free.

In the traditional way of teaching, the teacher had to check the image of each microscope individually to make sure that everyone sees the needed object details. This setting left limited time for content, and students were mainly observers. A digital classroom employing the Zeiss Stemi 305 cam with Labscope supports an interactive and personalized learning atmosphere. The teacher can monitor all student microscopes from his/her iPad, iPhone, or PC while moving around the classroom and therefore support those students who really need help.

Expand your Knowledge of Microscopy with MSA Membership!

\section{Whether your primary focus is
in the biological or the physica sciences, MSA takes your knowledge to the next level!}

Members Receive:

- A personal subscription to MSA's official journal, Microscopy and Microanalysis, and MSA's popular bi-monthly magazine, Microscopy Today.

- Peer Networking through the Society's Focused Interest Groups and Local Affiliated Societies.

- MSA Awards Programs, Scholarships, Speaker Opportunities, and much more!

Join MSA Today!

For more information: visit www.microscopy.org

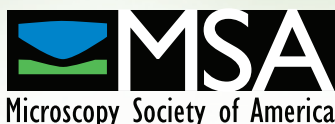

or call 1-800-538-3672 